$$
\begin{aligned}
& \text { طر احى قانون كنترل تعقيب مبتنى بر رويتكر بر اى كلاسى از سيستمهاى فازى جندجملهاى } \\
& \text { روزبه سليمى طارى '، على معرفيانيور }
\end{aligned}
$$

kurosh_st@yahoo.com، فارغالتحصيل كارشناسى ارشد، گروه كنترل، دانشكده مهندسى برق و كاميوتر، دانشگاه آزاد اسلامى واحد علوم و تحقيقات، تهران ، ايران ' استاديار، گروه كنترل، دانشكدة مهندسى برق و كامبيو تر، دانشگاه آزاد اسلامى واحد علوم و تحقيقات، تهران ، ايران،

$$
\begin{aligned}
& \text { جُكيده: در اين مقاله قانون كنترل تعقيب براى كلاسى از سيسم هاى فازى جندجمله اى طر احى مى شود. قانون كنترل از دو بخش رويتخر } \\
& \text { و فيدبك حالت تشكيل شده است. با استفاده از يك رويتگر فازى جندجمله ای، بردار حالت سيستم تخمين زده مىشود و بهره فيدبك } \\
& \text { جندجملهاى، از بردار حالت تخمين زده شده براى تحقق قانون كنترل استفاده مى كند. قانون كنترل فازى جندجملهاى، بردار حالت فرايند را به } \\
& \text { تعقيب از بردار حالت يكك مدل مرجع پيايدار تحت شاخص نرم بينهايت وادار مى كند. شرايط كافى براى تعيين پارامترهاى قانون كنترل در قالب } \\
& \text { يكك برنامه مجموع مربعات ارائه خو اهد شد. براى نشان دادن كارايى روش ارائه شده طراحى قانون كنترل و شبيه سازى در قالب جند مثال انجام } \\
& \text { مىشود. }
\end{aligned}
$$

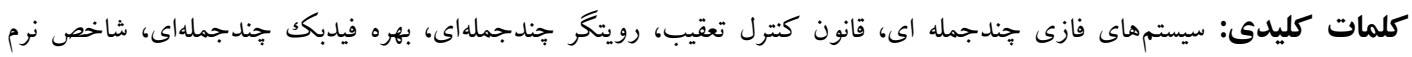

$$
\begin{aligned}
& \text { بى نهايت، مجموع مربعات. }
\end{aligned}
$$

\title{
Observer-Based Tracking Control Design for a Class of Fuzzy Polynomial Systems
}

\section{Roozbeh Salimi Tari, Ali Moarefianpour}

Abstract: In this paper tracking control law design for a class of polynomial fuzzy systems is considered. The control law consists of an observer and a state feedback. A polynomial fuzzy observer estimates the state vector of the plant, and then the estimated state vector is employed by a polynomial feedback gain to fulfill the control law. The polynomial fuzzy control law leads the state vector of the plant to track the state vector of a stable reference model subject to an $H_{\infty}$ performance. Sufficient conditions for determination of the control law parameters will be presented in the form of an SOS program. Additionally simulation results are presented to show the merits of the proposed control design approach.

Keywords: Polynomial fuzzy systems, tracking control law, polynomial observer, polynomial feedback gain, $H_{\infty}$ performance, sum of squares (SOS). 
شناسايى بديده رسوب در يك دستكاه تبادل حرارتى استفاده شده است.

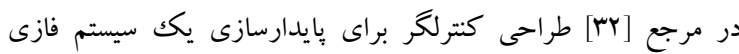

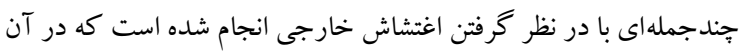

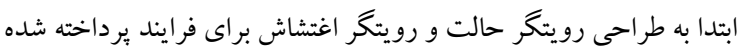
و سبس قانون كنترل يايدارساز براى سيستم حلقه بسته طراحى شده است.

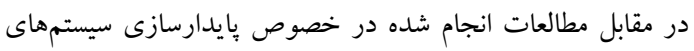

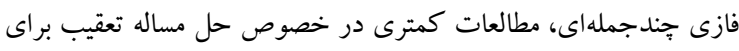

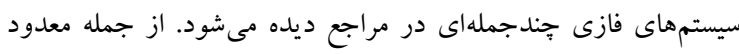

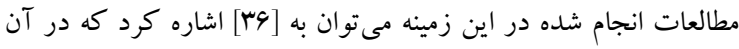

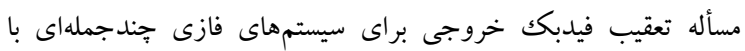

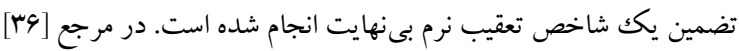

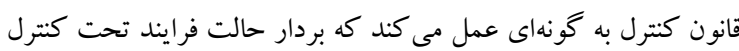

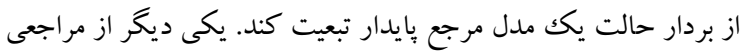

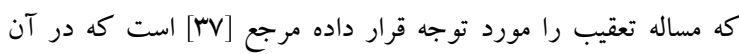

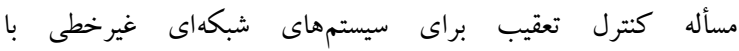

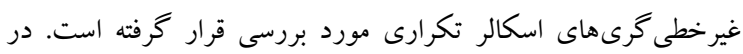

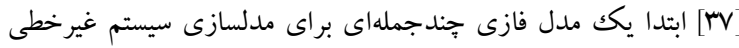

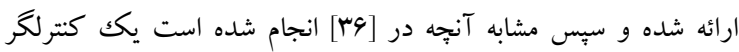

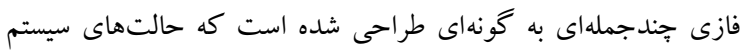

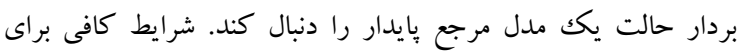

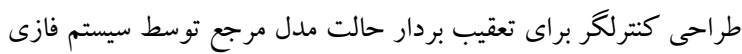

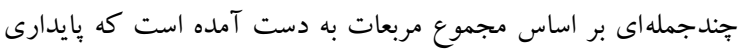
اتفاقى' و شرط كارايى نرم بىنهايت را نيز تضمين مى كند.

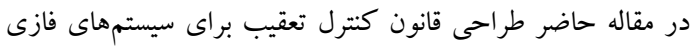

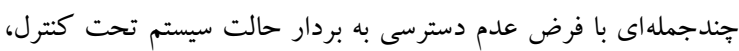

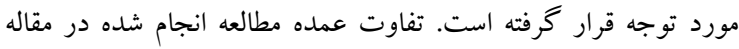

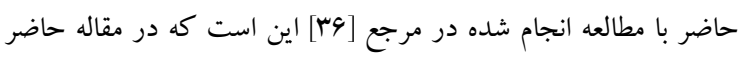

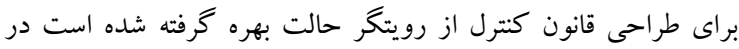

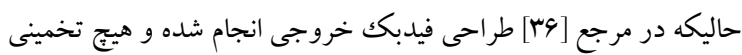

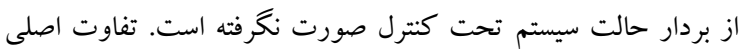

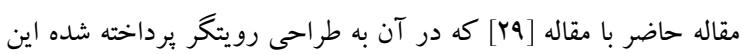

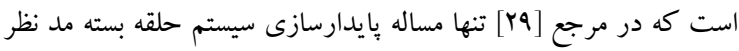

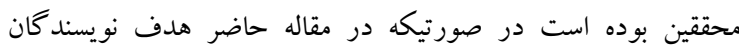

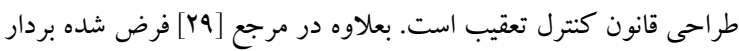

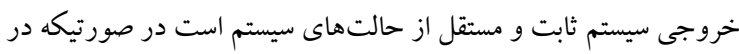
مقاله حاضر ماتريس خروجى به طور كلى وابسته به بردار حالت سيستم

$$
\text { در نظر كر فته شده است. }
$$

در بخش Y مقدمات رياضى مورد نياز ارائه مى مشوند. معرفى مدل

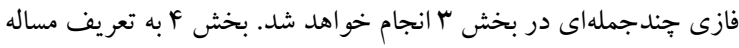

\footnotetext{
${ }^{1}$ Stochastic stability
}

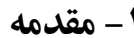

پايدارسازى و تعقيب همواره به صورت دو مسأله مهم در كنترل سيستمهاى غير خطى مورد توجه محققين بوده است. در سالهاى اخير

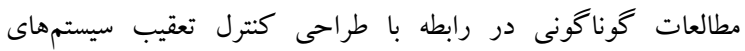

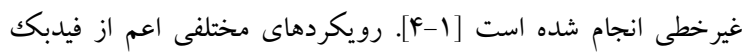

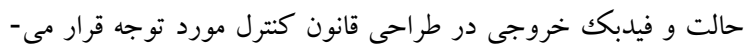

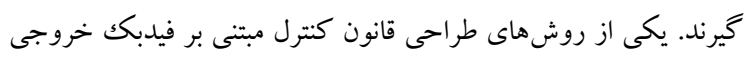
كه در طر احى قانون كنترل بايدارساز و همجنين تعقيب مورد استفاده قرار

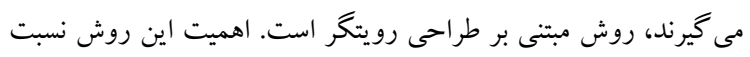

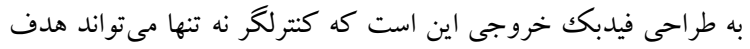

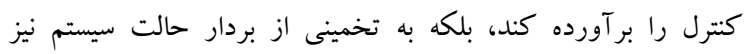
دستر سى دارد. يك دسته از سيستمهاى فازى كه مطالعات زيادى بر روى آن انجام

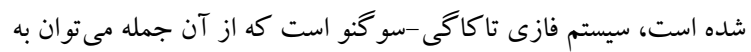

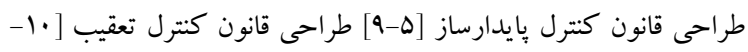

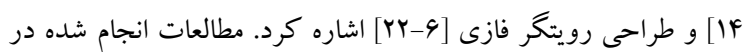

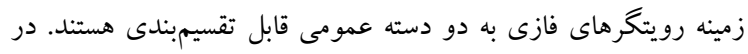

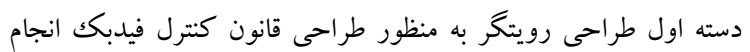

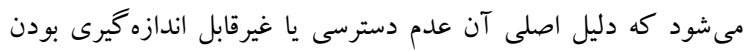

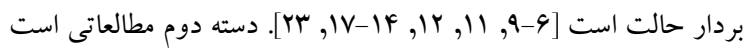

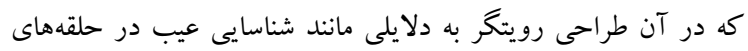

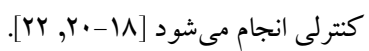
در سالهاى اخير دستهاى از سيستمهاى فازى مورد توجه محققين

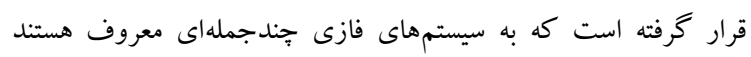

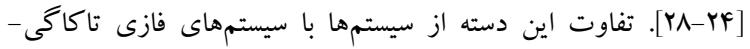

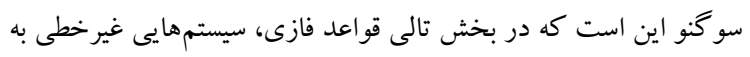

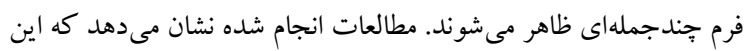

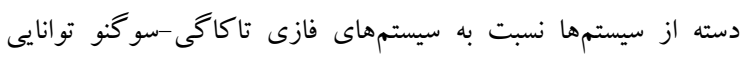
بهترى در توصيف سيستمهاى غيرخطى دارند [YY-YY]. ارائه شرايط

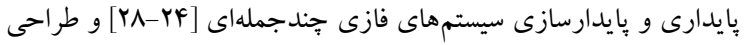

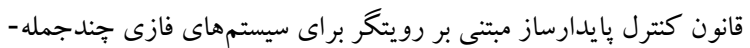

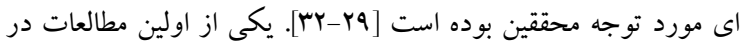

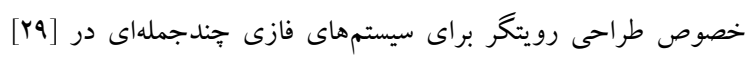

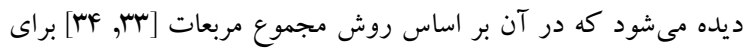

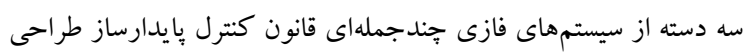

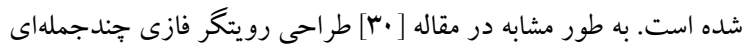
براى سيستمهاى غير خطى كه در معرض يكك اغتشاش خارجى محدود

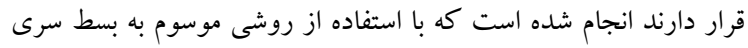

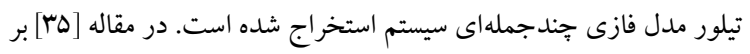

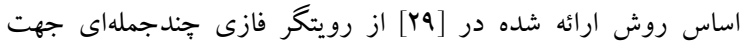




$$
\begin{aligned}
& {\left[\begin{array}{cc}
A & \frac{1}{\sqrt{2}}\left(-B+C^{T}\right) \\
\frac{1}{\sqrt{2}}\left(-B^{T}+C\right) & -D
\end{array}\right] \leq 0} \\
& A-B D^{-1} C \leq 0 \\
& \text { اثبات-با استفاده از لم مكمل شور مىتوان كفت: } \\
& {\left[\begin{array}{cc}
A & \frac{1}{\sqrt{2}}\left(-B+C^{T}\right) \\
\frac{1}{\sqrt{2}}\left(-B^{T}+C\right) & -D
\end{array}\right] \leq 0} \\
& A+\frac{1}{2}\left(-B+C^{T}\right) D^{-1}\left(-B+C^{T}\right)^{T} \\
& =A+\frac{1}{2} B D^{-1} B^{T}-\frac{1}{2} B D^{-1} C \\
& -\frac{1}{2} C^{T} D^{-1} B^{T}+\frac{1}{2} C^{T} D^{-1} C \leq 0
\end{aligned}
$$

بنابراين

$A-\frac{1}{2}\left(B D^{-1} C+C^{T} D^{-1} B^{T}\right)$

$$
\leq-\frac{1}{2} C^{T} D^{-1} C-\frac{1}{2} B D^{-1} B^{T}
$$

با توجه به اينكه سمت راست نامساوى اخير مثبت نيست بنابراين اثبات

تمام است.

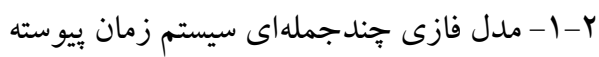

يكك سيسم فازى با r قاعده را در نظر بخيريد كه قاعده i ام آن به صورت

If $z_{1}(t)$ is $M_{i 1}$ and $\ldots$ and $z_{p}(t)$ is $M_{i p}$

Then $\dot{x}(t)=A_{i}(x(t)) x(t)+B_{i}(x(t)) u(t)$

$$
i=1,2, \ldots, r
$$

تعريف مىشود ([1]]). همانطور كه ملاحظه مىشود بخش تالى سيستم

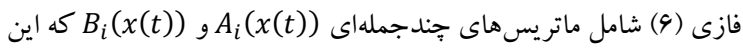

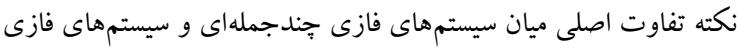

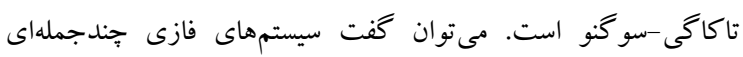

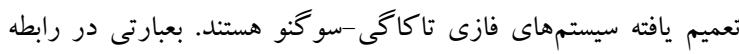
(9) اخر ماتريسهاى

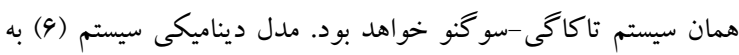

$$
\begin{gathered}
\dot{x}(t)=\sum_{i=1}^{r} h_{i}(z(t))\left\{A_{i}(x(t)) x(t)+B_{i}(x(t)) u(t)\right\} \\
y(t)=\sum_{i=1}^{r} h_{i}(z(t)) C_{i}(x(t)) x(t)
\end{gathered}
$$

اختصاص دارد. در بخش ه شرايط طراحى قانون كنترل در قالب يك

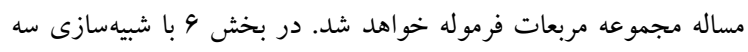

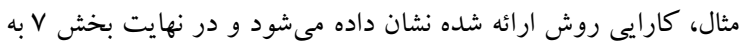
نتيجه كيرى از بحث اختصاص دارد.

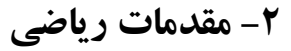

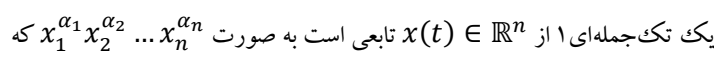

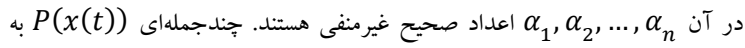

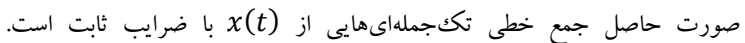
جندجملهاى (x)

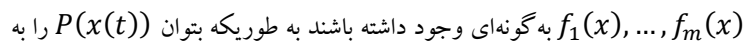
صورت ماتريس مربعى باشد عبارت

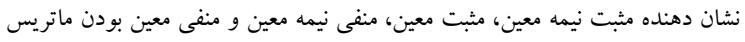
M هستند. همجنين $M$

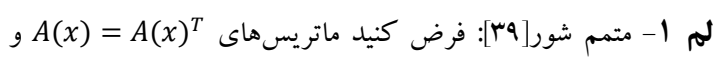

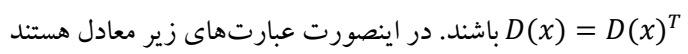
$\left[\begin{array}{cc}A(x) & B(x) \\ B(x)^{T} & D(x)\end{array}\right]<0 \quad-$ الف $A(x)<0, A(x)-B(x) D(x)^{-1} B(x)^{T}<0 \quad-ب$ $D(x)<0, D(x)-B(x)^{T} A(x)^{-1} B(x)<0 \quad-$

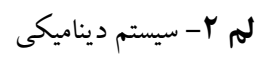

$$
\dot{x}=f(x, \omega)
$$

را در نظر بخيريد كه در آن x بردار حالت سيستم و ف يك سيخنال

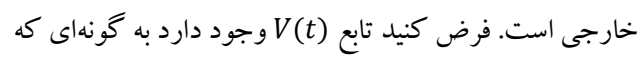

$$
V(t)>0
$$

$$
\dot{V}(t) \leq-x^{T} x+\sigma^{2} \omega^{T} \omega
$$

كه در آن م يك مقدار تعيين شده است، در اينصورت بهره انرزى سيستم از ورودى $\omega$ تا خروجى $\tilde{x}$ محدود است. اثبات- با انتخر الخيرى از طرفين عبارت دوم رابطه (r) نسبت به زمان و با فرض پايدارى سيستم مىتوان نوشت:

$$
\int_{0}^{\infty} x^{T} x \mathrm{~d} t-V(0) \leq \sigma^{2} \int_{0}^{\infty} \omega^{T} \omega \mathrm{d} t
$$

رابطه اخير بيان كننده محدود بودن بهره انرزى سيستم يا همان معيار

نرم بىنهايت است. لهم ب- فرض كنيد ماتريسهاى 0 > A، 0 > D و ماتريسهاى B

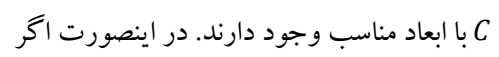

${ }^{1}$ Monomial 
بهترتيب ماتريس سيستم، ماتريس ورودى و ماتريس خروجى مدل مرجع

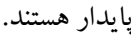
دياكرام بلو كى سيستم حلقه بسته در شكل انشان داده شده است.

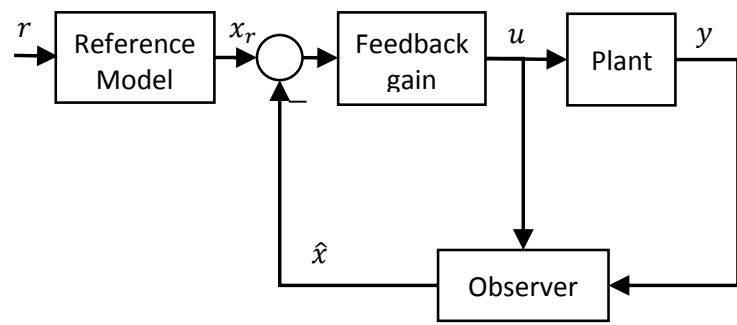
شكل ا: دياگر ام بلو كى سيستم حلقه بسته

\section{ع- طراحى قانون كنترل مبتنى بر رويتكر}

در اين قسمت شرايط طراحى قانون كنترل تعقيب براى سيستم (A) به

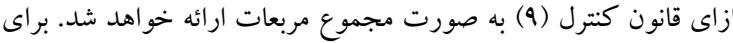

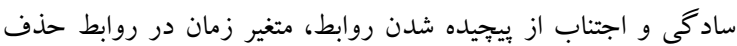
مىشود. بعبارتى (t) به صورت X نشان داده خواهد شد. همانطور كه

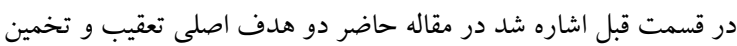
بردار حالت سيستم به طور همزمان مورد توجه است. براى اين منظور

$$
\begin{aligned}
& \text { خطاى تخمين بردار حالت سيستم، به صورت } \\
& e=x-\hat{x}
\end{aligned}
$$

تعريف مىشود. بر اساس رابطه اخير و با استناد به رابطههاى (1) و ( •) ديناميك خطاى تخمين به صورت

$$
\begin{aligned}
\dot{e}=\dot{x}-\dot{\hat{x}}=\sum_{i=1}^{r} \sum_{j=1}^{r} & h_{i}(z) h_{j}(z)\left[\left[A_{i}(x) x+B_{i} u\right]\right. \\
& -\left[A_{i}(\hat{x}) \hat{x}+B_{i} u\right. \\
& \left.\left.+L_{i}(\hat{x})\left(C_{j}(x) x-C_{j}(\hat{x}) \hat{x}\right)\right]\right]
\end{aligned}
$$

$$
\begin{aligned}
=\sum_{i=1}^{r} \sum_{j=1}^{r} h_{i}(z) h_{j}(z) & {\left[\left(A_{i}(x) x-A_{i}(\hat{x}) \hat{x}\right.\right.} \\
- & \left.L_{i}(\hat{x})\left(C_{j}(x) x-C_{j}(\hat{x}) \hat{x}\right)\right]
\end{aligned}
$$

قابل استخراج است. با تغيير متغير

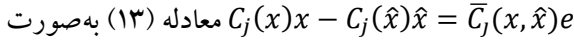

$$
\dot{e}=\sum_{i=1}^{r} \sum_{j=1}^{r} h_{i}(z) h_{j}(z)\left(\bar{A}_{i}(x, \hat{x})-L_{i}(\hat{x}) \bar{C}_{J}(x, \hat{x})\right) e
$$

قابل بيان است. رابطه اخير ديناميك خطاى تخمين را توصيف مى كند كه در آن (t) حندجملهاى از بردار حالت رويتخر باشد.
قابل نمايش است كه در آن $x(t) \in \mathbb{R}^{n}$ بردار حالت، $u(t) \in \mathbb{R}^{m}$ ورودى، ك $C_{i}(x(t)) \in \mathbb{R}^{q \times n}, B_{i}(x(t)) \in \mathbb{R}^{n \times m}$ ، $A_{i}(x(t)) \in \mathbb{R}^{n \times n}$ ماتريسهاى جندجملهاى از x هستند، به ترتيب ماتريس سيستم، ماتريس

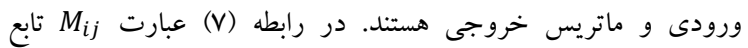
عضويت متناظر با $i$ امين قانون و ز امين متغير مقدم و (t)

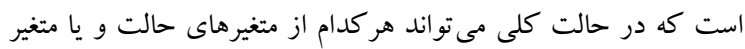

$$
\text { قابل اندازه گيرى خارج از سيستم باشد. }
$$

\section{"r- تعريف مساله}

در اين مقاله دسته خاصى از سيستمهاى فازى جندجملهاى مورد

$$
\begin{gathered}
\dot{x}(t)=\sum_{i=1}^{r} h_{i}(z(t))\left\{A_{i}(x(t)) x(t)+B_{i}(\zeta(t)) u(t)\right\} \\
y(t)=\sum_{i=1}^{r} h_{i}(z(t)) C_{i}(x(t)) x(t)
\end{gathered}
$$

نشان داده مىشوند. در رابطه (^) ماتريس سيستم تابعى از بردار حالت

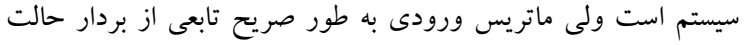

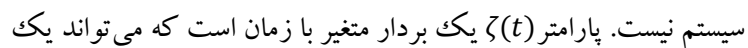
متغير خارجى قابل اندازهگيرى باشد. براى وادار كردن بردار حالت سيستم (A) به ييروى از بردار حالت يكك

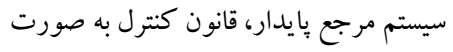

$$
u(t)=\sum_{j=1}^{r} h_{j}(z(t)) K_{j}(\hat{x}(t))\left(\hat{x}(t)-x_{r}(t)\right)
$$

در نظر گرفته مى شود كه در آن (t)

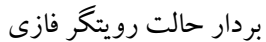

$$
\begin{gathered}
\dot{\hat{x}}(t)=\sum_{i=1}^{r} h_{i}(z(t)) \begin{array}{c}
\left\{A_{i}(\hat{x}(t)) \hat{x}(t)+B_{i}(\zeta(t)) u(t)\right. \\
\left.+L_{i}(\hat{x}(t))(y(t)-\hat{y}(t))\right\}
\end{array} \\
\hat{y}(t)=\sum_{i=1}^{r} h_{i}(z(t)) C_{i}(\hat{x}(t)) \hat{x}(t)
\end{gathered}
$$

است. بعبارتى

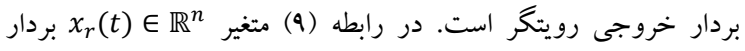
حالت مدل مرجع بايدار

$$
\begin{gathered}
\dot{x}_{r}(t)=A_{r} x_{r}(t)+B_{r} r(t) \\
y_{r}(t)=C x_{r}(t)
\end{gathered}
$$

است كه در آن

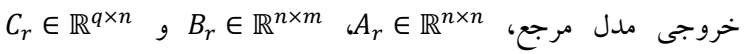


را به عنوان كانديداى تابع ليإيانوف در نظر بحيريد كه در آن

$$
\begin{aligned}
& X=\left[\begin{array}{ccc}
X_{11} & 0 & 0 \\
0 & X_{22} & 0 \\
0 & 0 & X_{33}
\end{array}\right] \\
& \text { مشتق زمانى تابع (t) ل در امتداد مسير حالتهاى سيستم به صورت }
\end{aligned}
$$

$$
\begin{aligned}
\dot{V}(t)= & \dot{\tilde{x}}^{T} X \tilde{x}+\tilde{x}^{T} X \dot{\tilde{x}} \\
= & \sum_{i=1}^{r} \sum_{j=1}^{r} h_{i}(z) h_{j}(z)\left\{\tilde{x}^{T} \tilde{A}_{i j}{ }^{T} X \tilde{x}+r^{T} \widetilde{E}_{l}{ }^{T} X \tilde{x}\right. \\
& \left.+\tilde{x}^{T} X \tilde{A}_{i j} \tilde{x}+\tilde{x}^{T} X \widetilde{E}_{l} r\right\} \\
=\sum_{i=1}^{r} \sum_{j=1}^{r} h_{i}(z) & h_{j}(z) \tilde{x}^{T}\left(\tilde{A}_{i j}{ }^{T} X+X \tilde{A}_{i j}\right) \tilde{x} \\
& +r^{T} \widetilde{E}_{l}^{T} X \tilde{x}+\tilde{x}^{T} X \widetilde{E}_{l} r-r^{T} \sigma^{2} r \\
& +r^{T} \sigma^{2} r \\
&
\end{aligned}
$$$$
\dot{V}(t)=\sum_{i=1}^{r} \sum_{j=1}^{r} h_{i}(z) h_{j}(z) \Phi^{T} \Psi_{i j} \Phi-\tilde{x}^{T} \tilde{x}+\sigma^{2} r^{T} r
$$

بازنويسى كرد كه در آن

$$
\begin{gathered}
\Psi_{i j}=\left[\begin{array}{cc}
\tilde{A}_{i j}{ }^{T} X+X \tilde{A}_{i j}+I & X \widetilde{E}_{l} \\
\widetilde{E}_{l}^{T} X & -\sigma^{2} I
\end{array}\right] \\
\Phi=\left[\begin{array}{l}
\tilde{x} \\
r
\end{array}\right]
\end{gathered}
$$

و $\sigma$ اسكالرى است كه بايد توسط طراح تعيين شود. از آنجايى كه در رابطه (r) بايد 0 (r) صور تيكه 0 ك

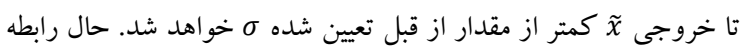
X نشان داد

$$
\begin{aligned}
& \Psi_{i j}=\left[\begin{array}{c}
\mathcal{L}\left\{X_{11}\left(A_{i}^{x}+B_{i} K_{j}\right)\right\} \\
-\left(B_{i} K_{j}\right)^{T} X_{11} \\
\left(A_{i}^{x}-A_{r}\right)^{T} X_{11} \\
-B_{r}^{T} X_{11}
\end{array}\right.
\end{aligned}
$$

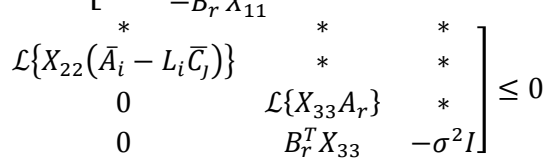

رابطه اخير بدليل ظاهر شدن ضرب بارامترهاى مجهول در يكديخر غير باري

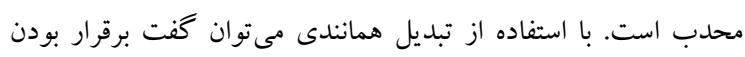

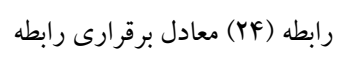

$$
\chi^{T} \Psi_{i j} \chi \leq 0
$$

است كه در آن لdiag $\left(X_{11}^{-1}, I, I, I\right)$. صورت
براى اينكه قانون كنترل، اهداف بايدارسازى و تعقيب را به طور

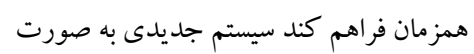

$$
\dot{\tilde{x}}=\sum_{i=1}^{r} h_{i}(z) \sum_{j=1}^{r} h_{j}(z)\left[\tilde{A}_{i j} \tilde{x}+\tilde{E}_{i} r\right]
$$

$$
\text { در نظر كر فته مى شود كه در آن }
$$

$\tilde{A}_{i j}$

$$
\begin{gathered}
=\left[\begin{array}{ccc}
A_{i}(x)+B_{i}(\zeta) K_{j}(\hat{x}) & -B_{i}(\zeta) K_{j}(\hat{x}) & A_{i}(x)-A_{r} \\
0 & \bar{A}_{i}-L_{i}(\hat{x}) \bar{C}_{j} & 0 \\
0 & 0 & A_{r}
\end{array}\right](\text { (1) } \\
\tilde{x}=\left[\begin{array}{c}
x-x_{r} \\
e \\
x_{r}
\end{array}\right], \widetilde{E}_{l}=\left[\begin{array}{c}
-B_{r} \\
0 \\
B_{r}
\end{array}\right]
\end{gathered}
$$

كه در آن، 1- سيستم (10) را در نظر بكيريد. اكر ماتريسهاى ح

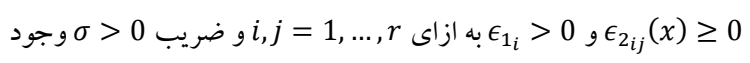
داشته باشند به كونهاى كه

$$
\left[\begin{array}{ccc}
X_{11} & 0 & 0 \\
0 & X_{22} & 0 \\
0 & 0 & X_{33}
\end{array}\right]-\epsilon_{1 i} I \text { is SOS }
$$

$$
-\left(\Omega_{i j}(x)+\epsilon_{2 i j}(x) I\right) \text { is SOS }
$$

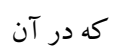

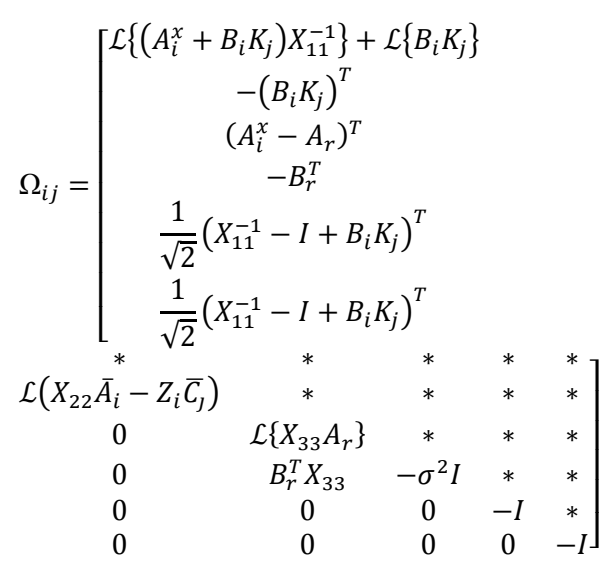

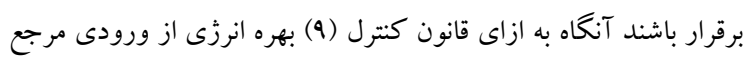

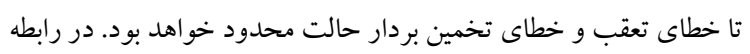

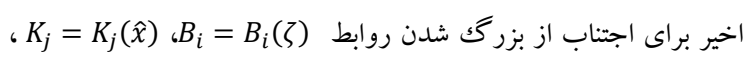
در نظر $Z_{i}=Z_{i}(\hat{x})=X_{22} L_{i}(\hat{x}) ， A_{i}^{x}=A_{i}(x) \quad A_{i}=A_{i}(\hat{x})$

$$
\text { كرفته شده است. }
$$$$
\text { اثبات- تابع }
$$

$$
V(t)=\tilde{x}^{T} X \tilde{x}
$$


اثبات- از آنجاييكه هدف تنها تخمين بردار حالت سيستم (A) است

$$
\text { بنابر اين تابع ليايانوف به صورت البه }
$$

$V(t)=e^{T} P e$

در نظر گرفته مى شود كه در آن

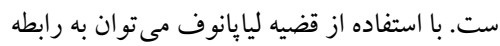
$\dot{V}(t)=\dot{e}^{T} X e+e^{T} P \dot{e}$

$$
\begin{array}{r}
=\sum_{i=1}^{r} \sum_{j=1}^{r} h_{i}(z) h_{j}(z) e^{T}\left[\left(\bar{A}_{i}-L_{i} \bar{C}_{j}\right)^{T} P\right. \\
\left.+P\left(\bar{A}_{i}-L_{i} \bar{C}_{j}\right)\right] e \leq 0
\end{array}
$$

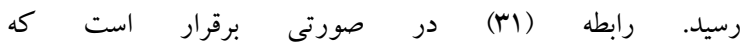
曰 $.\left(\bar{A}_{i}-L_{i} \bar{C}_{j}\right)^{T} P+P\left(\bar{A}_{i}-L_{i} \bar{C}_{j}\right) \leq 0$

قضيه r- حالتى را در نظر بخيريد كه در آن

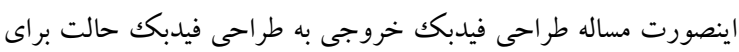

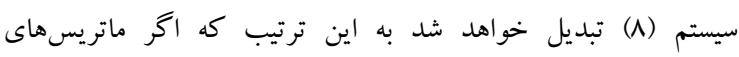

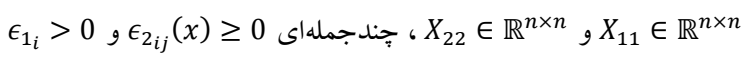
به ازاى r, r,

$$
\begin{gathered}
{\left[\begin{array}{cc}
X_{11} & 0 \\
0 & X_{22}
\end{array}\right]-\epsilon_{1_{i}} I \text { is SOS }} \\
-\left(\Omega_{i j}(x)+\epsilon_{2 i j}(x) I\right) \text { is SOS }
\end{gathered}
$$

برقرار باشد كه در آن

$$
\Omega_{i j}(x)=\left[\begin{array}{ccc}
\mathcal{L}\left\{A_{i}^{x} X_{11}^{-1}+B_{i} Z_{j}\right\} & * & * \\
\left(A_{i}^{x}-A_{r}\right)^{T} & \mathcal{L}\left\{X_{22} A_{r}\right\} & * \\
-B_{r}^{T} & B_{r}^{T} X_{22} & -\sigma^{2} I
\end{array}\right]
$$

و آنگاه با قانون كنترل

$$
u=\sum_{j=1}^{r} h_{j}(z)\left[K_{j}(x)\left(x-x_{r}\right)\right]
$$

بهره انرزى سيستم حلقه بسته از ورودى مرجع تا خروجى خطاى تعقيب محدود است.

اثبات- اثبات تا حد زيادى مشابه اثبات قضيه ا است با اين تفاوت كه بايد x=x در نظر گرفته شود. براى فراهم كردن همزمان شران شرايط

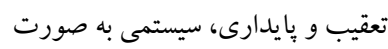

$$
\dot{\tilde{x}}=\sum_{i=1}^{r} h_{i}(z) \sum_{j=1}^{r} h_{j}(z)\left[\tilde{A}_{i j} \tilde{x}+\tilde{E}_{i} r\right]
$$

در نظر گرفته مىشود كه در آن

$$
\begin{aligned}
& {\left[\mathcal{L}\left\{\left(A_{i}^{x}+B_{i} K_{j}\right) X_{11}^{-1}+B_{i} K_{j}-B_{i} K_{j}\right\}\right.} \\
& -\left(B_{i} K_{j}\right)^{T} \\
& \left(A_{i}^{x}-A_{r}\right)^{T}
\end{aligned}
$$

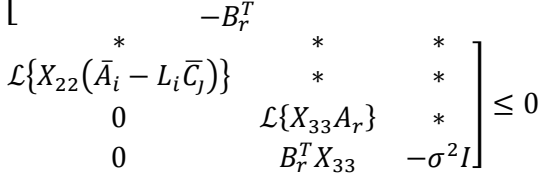

بازنويسى كرد كه در آن رابطه اخير معادل

$$
\begin{aligned}
& {\left[\begin{array}{cc}
\mathcal{L}\left\{A_{i}^{x} X_{11}^{-1}+B_{i} K_{j}\right\} & * \\
-\left(B_{i} K_{j}\right)^{T} & \mathcal{L}\left\{X_{22}\left(\bar{A}_{i}-L_{i} \bar{C}_{J}\right)\right\} \\
\left(A_{i}^{x}-A_{r}\right)^{T} & 0 \\
-B_{r}^{T} & * \\
* & * \\
* & * \\
\mathcal{L}\left\{X_{33} A_{r}\right\} & * \\
B_{r}^{T} X_{33} & -\sigma^{2} I
\end{array}\right]-\left[\begin{array}{cc}
X_{11}^{-1}-I & B_{i} K_{j} \\
0 & 0 \\
0 & 0 \\
0 & 0
\end{array}\right]\left[\begin{array}{cc}
-I & 0 \\
0 & -I
\end{array}\right]} \\
& {\left[\begin{array}{cccc}
\left(B_{i} K_{j}\right)^{T} & 0 & 0 & 0 \\
X_{11}^{-1}-I & 0 & 0 & 0
\end{array}\right] \leq 0}
\end{aligned}
$$

است. ماتريس ظاهر شده در سمت جِّ رابطه اخير متقارن است. با توجه

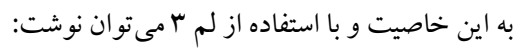

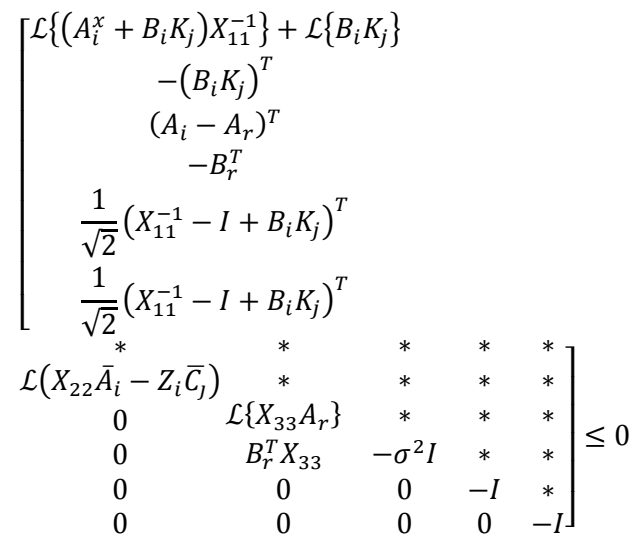

نتيجه فرعى 1- در صورتيكه تنها طراحى رويتخر مد نظر باشد، اكر

$$
\begin{gathered}
\left(\bar{A}_{i}-L_{i} \bar{C}_{\jmath}\right)^{T} P+P\left(\bar{A}_{i}-L_{i} \bar{C}_{\jmath}\right) \leq 0 \\
i=1, \ldots, r \\
j=1, \ldots, r
\end{gathered}
$$

برقرار باشد كه در آن P يكك ماتريس مثبت معين است، آنگاه رويتگر

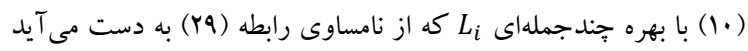

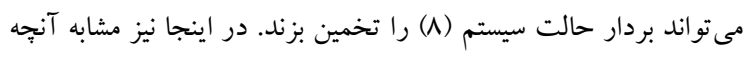
در اثبات قضيه 1 بيان شد في . $L_{i}=L_{i}(\hat{x})$ 
در همه مثالها از جعبه ابزار يالميب' در متلب براى به دست آوردن

$$
\text { يار امترهاى كنترلكر استفاده شده است. }
$$

- 1- مثال

$$
\left\{\begin{array}{c}
\dot{x}_{1}=10\left(x_{2}-x_{1}\right)+u_{1} \\
\dot{x}_{2}=28 x_{1}-x_{2}-x_{1} x_{3}+u_{2} \\
\dot{x}_{3}=x_{1} x_{2}-\frac{8}{3} x_{3}+u_{3}
\end{array}\right.
$$$$
\text { سيستم آشوبناك لورنز رادر نظر بحيريد ([F]) }
$$

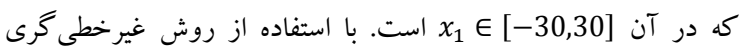

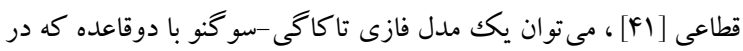
آن متغير مقدم Z (F) را در بازه تعريف شده را به طور دقيق توصيف كند. بِارامترهاى

$$
\text { سيستم فازى به صورت }
$$

$$
\begin{aligned}
& A_{1}=\left[\begin{array}{ccc}
-10 & 10 & 0 \\
28 & -1 & -30 \\
0 & 30 & -\frac{8}{3}
\end{array}\right], A_{2}=\left[\begin{array}{ccc}
-10 & 10 & 0 \\
28 & -1 & 30 \\
0 & -30 & -\frac{8}{3}
\end{array}\right] \\
& B_{1}=B_{2}=\left[\begin{array}{lll}
1 & 0 & 0 \\
0 & 1 & 0 \\
0 & 0 & 1
\end{array}\right], C_{1}=C_{2}=\left[\begin{array}{lll}
1 & 0 & 0
\end{array}\right] \\
& h_{1}(x)=\frac{1}{2}\left(1+\frac{x_{1}}{30}\right), h_{2}(x)=\frac{1}{2}\left(1-\frac{x_{1}}{30}\right) \\
& \text { بدست مى آيند. با انتخاب بارامترهاى مدل مرجع به صورت } \\
& A_{r}=\left[\begin{array}{ccc}
0 & 1 & 0 \\
0 & 0 & 1 \\
-2 & -4 & -3
\end{array}\right], B_{r}=\left[\begin{array}{lll}
1 & 0 & 0 \\
0 & 1 & 0 \\
0 & 0 & 1
\end{array}\right] \text {, } \\
& C_{r}=\left[\begin{array}{lll}
1 & 0 & 0
\end{array}\right]
\end{aligned}
$$

و با انتخاب $5=5$ متغيرهاى تصميم گيرى و پارامترهاى كنترلخر و

$$
\text { رويتخر به صورت ابتخاب }
$$$$
\begin{gathered}
X_{11}=10^{-7}\left[\begin{array}{ccc}
0.1369 & -0.3255 & -0.0018 \\
-0.3255 & 0.9082 & 0.0003 \\
-0.0018 & 0.0003 & 0.6679
\end{array}\right], \\
X_{22}=\left[\begin{array}{ccc}
0.4325 & -0.2616 & 0 \\
-0.2616 & 0.2748 & 0 \\
0 & 0 & 0.247
\end{array}\right] \\
X_{33}=\left[\begin{array}{ccc}
0.0114 & -0.0133 & 0.0266 \\
-0.0133 & 0.0114 & 0.0398 \\
0.0266 & 0.0398 & 0.0513
\end{array}\right] \\
K_{1}=\left[\begin{array}{ccc}
-6457.481 & 492.268 & -0.729 \\
787.778 & -6895.572 & 0.188 \\
-0.182 & 0.517 & -5747.302
\end{array}\right] \\
K_{2}=\left[\begin{array}{ccc}
-6451.520 & 492.440 & -0.381 \\
787.216 & -6895.329 & 0.404 \\
-0.498 & 0.0935 & -5747.282
\end{array}\right]
\end{gathered}
$$

$$
L_{1}=\left[\begin{array}{l}
44.704 \\
61.498 \\
18.582
\end{array}\right], L_{2}=\left[\begin{array}{c}
44.704 \\
61.497 \\
-18.582
\end{array}\right]
$$

بدست مى آيند. نتايج شبيهسازى سيستم حلقه بسته را به ازاى انتخاب شرايط اوليه سيستم اصلى به صورت شرايط اوليه رويتخر به صورت

$$
\begin{gathered}
\tilde{A}_{i j}=\left[\begin{array}{cc}
A_{i}^{\chi}+B_{i} K_{j} & A_{i}^{x}-A_{r} \\
0 & A_{r}
\end{array}\right] \\
\tilde{x}=\left[\begin{array}{c}
x-x_{r} \\
x_{r}
\end{array}\right], \widetilde{E}_{\iota}=\left[\begin{array}{c}
-B_{r} \\
B_{r}
\end{array}\right]
\end{gathered}
$$

است. مانند آنجه در اثبات قضيه ا بيان شد با در نظر گرفتن تابع ليايانوف

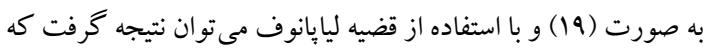
$\dot{V}(t)=\sum_{i=1}^{r} \sum_{j=1}^{r} h_{i}(z) h_{j}(z) \Phi^{T} \Psi_{i j} \Phi-\tilde{x}^{T} \tilde{x}+\sigma^{2} r^{T} r \leq 0$

$$
\text { كه در آن }
$$

$$
\Psi_{i j}=\left[\begin{array}{ccc}
\mathcal{L}\left\{X_{11}\left(A_{i}^{x}+B_{i} K_{j}\right)\right\} & * & * \\
\left(A_{i}^{x}-A_{r}\right)^{T} X_{11} & \mathcal{L}\left\{X_{22} A_{r}\right\} & * \\
-B_{r}^{T} X_{11} & B_{r}^{T} X_{22} & -\sigma^{2} I
\end{array}\right] \leq 0
$$

است. مانند آنجه در اثبات قضيه ا ذكر شد برقرارى رابطه (N؟r) معادل برقرارى رابطه $\chi^{T} \Psi_{i j} \chi=\left[\begin{array}{ccc}\mathcal{L}\left\{\left(A_{i}^{x}+B_{i} K_{j}\right) X_{11}^{-1}\right\} & * & * \\ \left(A_{i}^{x}-A_{r}\right)^{T} & \mathcal{L}\left\{X_{22} A_{r}\right\} & * \\ -B_{r}^{T} & B_{r}^{T} X_{22} & -\sigma^{2} I\end{array}\right] \leq 0$ (rq) است كه در آن $\chi$ است. رابطه (ه) ) به دليل حاصلضرب دو يارامتر مجهول تغيير متغير

$$
\left[\begin{array}{ccc}
\mathcal{L}\left\{A_{i}^{x} X_{11}^{-1}+B_{i} Z_{j}\right\} & * & * \\
\left(A_{i}^{x}-A_{r}\right)^{T} & \mathcal{L}\left\{X_{22} A_{r}\right\} & * \\
-B_{r}^{T} & B_{r}^{T} X_{22} & -\sigma^{2} I
\end{array}\right] \leq 0
$$

$$
\text { قابل بازنو يسى است. }
$$

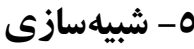

در اين بخش براى نشان دادن كارايى قضاياى ارائه شده در بخش

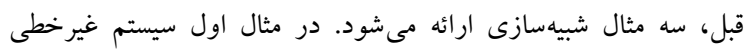

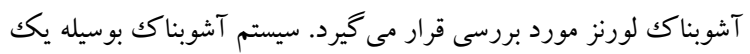

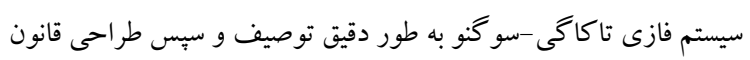

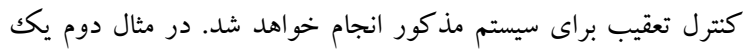

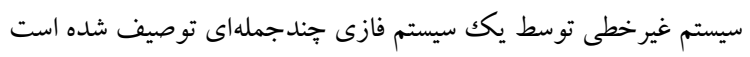

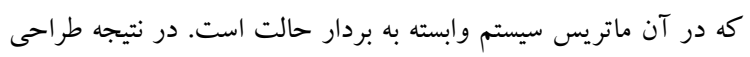

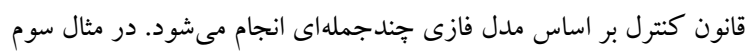

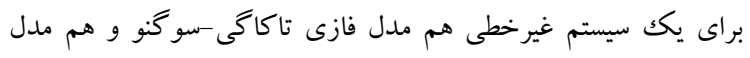
فازى جندجملهاى ارائه و طراحى قانون كنترل تعقيب براى هر دو دو سيستم

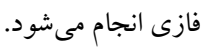




$$
\begin{gathered}
A_{1}(x)=\left[\begin{array}{cc}
1 & -0.3 x_{2} \\
-1.5 & -2-x_{2}^{2}
\end{array}\right], A_{2}(x) \\
=\left[\begin{array}{cc}
-0.2172 & -0.3 x_{2} \\
-1.5 & -2-x_{2}^{2}
\end{array}\right] \\
B_{1}(x)=B_{2}(x)=\left[\begin{array}{c}
x_{1}^{2}+1 \\
0
\end{array}\right], \quad C_{1}=C_{2}=\left[\begin{array}{ll}
1 & 0
\end{array}\right] \\
h_{1}(z)=\frac{\sin x_{1}+0.21727}{1.2172 x_{1}}, h_{2}(z)=\frac{x_{1}-\sin x_{1}}{1.2172 x_{1}}
\end{gathered}
$$

قابل استخراج است. نمودار فاز سيستم (FY) در شكل F نشان داده شده

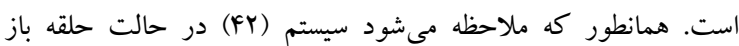
نإيايدار است.

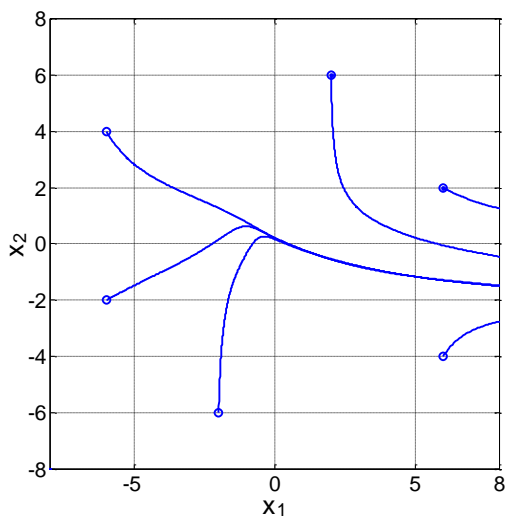

شكل f: نمو دار فاز سيستم حلقه باز مثال دوم

$$
\text { با انتخاب ماتريس هاى مدل مرجع بهصورت }
$$

$$
A_{r}=\left[\begin{array}{ll}
-1 & 0.5 \\
-2 & -4
\end{array}\right], B_{r}=\left[\begin{array}{c}
10 \\
0
\end{array}\right], C_{r}=\left[\begin{array}{ll}
1 & 0
\end{array}\right]
$$

و با انتخاب $\sigma=0.4$ متغيرهاى تصميم گيرى و بارامترهاى كنترلغر و رويتخر فازى جندجملهاى به صورت

$$
\begin{gathered}
X_{11}=10^{-3}\left[\begin{array}{cc}
-0.0064 & 0 \\
0 & 0.3557
\end{array}\right] \\
X_{22}=\left[\begin{array}{cc}
0.0799 & 0 \\
0 & 0.006
\end{array}\right] \\
X_{33}=10^{-4}\left[\begin{array}{ll}
0.0265 & 0.0097 \\
0.0097 & 0.2049
\end{array}\right] \\
K_{1}=\left[\begin{array}{ll}
-2.468 & -0.0593+0.0064 \hat{x}_{2}
\end{array}\right] \\
K_{2}=\left[\begin{array}{ll}
-4.051 & -0.515+0.318 \hat{x}_{2}
\end{array}\right] \\
L_{1}=\left[\begin{array}{c}
88.655-2.35 \times 10^{-12} \hat{x}_{2} \\
-4.415-1.824 \hat{x}_{2}
\end{array}\right] \\
L_{2}=\left[\begin{array}{c}
86.994-2.35 \times 10^{-12} \hat{x}_{2} \\
-4.428-1.838 \hat{x}_{2}
\end{array}\right]
\end{gathered}
$$

بدست مى آيند. نتايج شبيهسازى با انتخاب شرايط اوليه به صورت

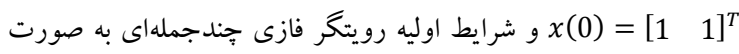
ملاحظك مـ ملاحظه مى شود سيستم حلقه بسته بايدار شده و تعقيب و تخمين بردار

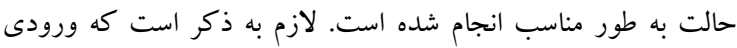

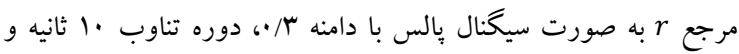
عرض بالس •هـ٪ در نظر خرفته شده است.
شكل r و شكل r مشاهده كرد. همانطور كه در شكل r ملاحظه مى شود قانون كنترل باعث شده كه تعقيب با دقت بالايى انجام شود. همجنين

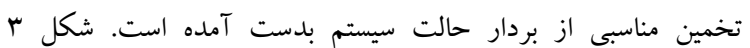

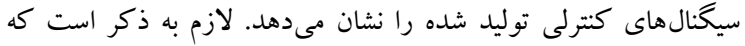

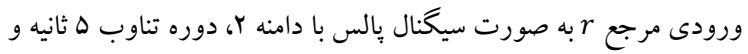
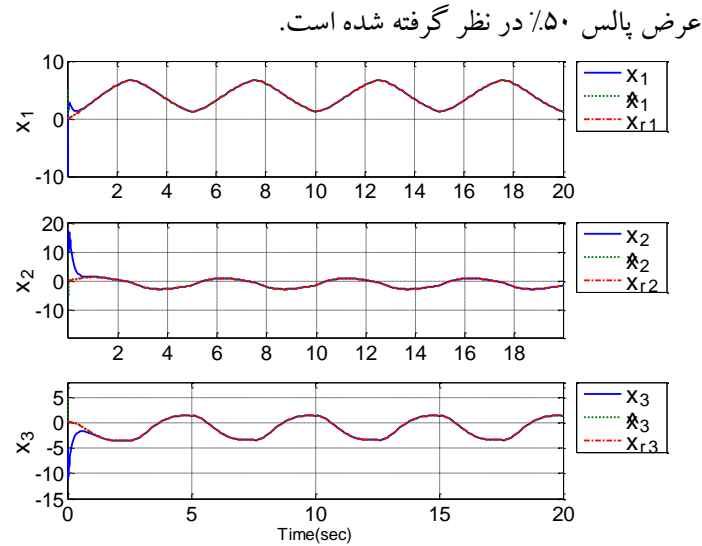

شكل r: نتايج تعقيب و تخمين همزمان سيستم مثال اول
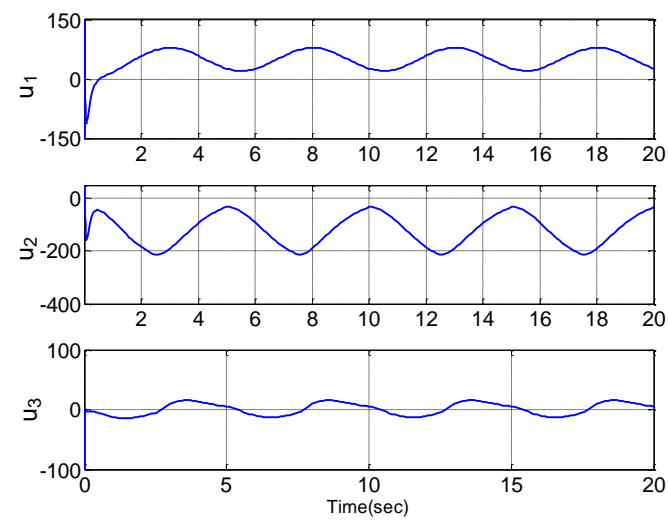

شكل rّ: قانون كنترل سيستم مثال اول

r

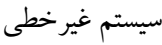

$$
\left\{\begin{array}{c}
\dot{x}_{1}=\sin x_{1}-0.3 x_{2}+\left(x_{1}{ }^{2}+1\right) u \\
\dot{x}_{2}=-1.5 x_{1}-2 x_{2}-x_{2}{ }^{3} \\
y=x_{1}
\end{array}\right.
$$

را در نظر بخيريد كه در آن متغير حالت 1 أبل ابل اندازهيرى است

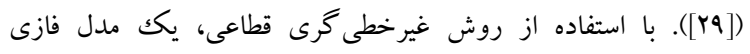

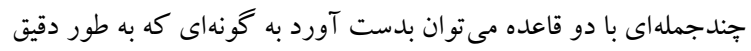
و جامع سيستم (FY) را توصيف كند. بردار متغير مقدم 1 (F) 1 مامل

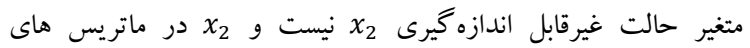
جندجملهاى كننده سيستم غير خطى (Fr) به صورت 


$$
\begin{gathered}
X_{33}=\left[\begin{array}{cc}
2.1787 & -0.1536 \\
-0.1536 & 1.3586
\end{array}\right] \\
K_{1}=\left[\begin{array}{ll}
-1.4982 & 0.2189
\end{array}\right], K_{2}=\left[\begin{array}{ll}
-1.5013 & 0.2243
\end{array}\right] \\
K_{3}=\left[\begin{array}{ll}
-1.5013 & 0.2243
\end{array}\right], K_{4}=\left[\begin{array}{ll}
-1.5013 & 0.2243
\end{array}\right] \\
L_{1}=\left[\begin{array}{c}
3.2682 \\
-0.9292
\end{array}\right], L_{2}=\left[\begin{array}{c}
3.2295 \\
-1.0764
\end{array}\right], \\
L_{3}=\left[\begin{array}{c}
3.1668 \\
-0.8591
\end{array}\right], L_{4}=\left[\begin{array}{c}
3.1666 \\
-1.0586
\end{array}\right]
\end{gathered}
$$

بدست مى آيند. نتايج شبيهسازى درشكل 9 نشان داده شده است كه در آن شرايط اوليه سيستم به صورت رويتخر فازى به صورت

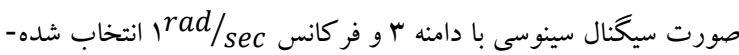

ند.
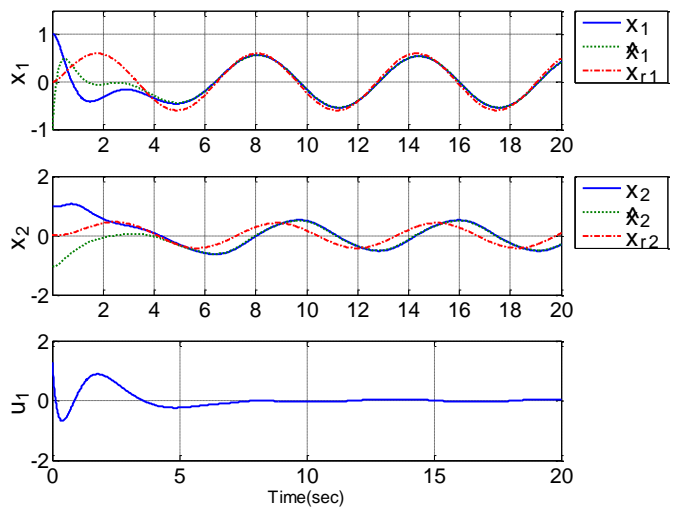

شكل צ: نتايج شبيه سازى مثال سوم براى مدل تاكاحى-سو كنو

حال به طراحى قانون كنترل بر اساس مدل فازى جندجملهاى برداخته

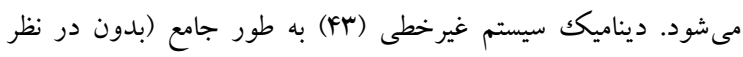
كرفتن بازه تغييرات براى حالت نمايش است كه در آن

$$
\begin{gathered}
A_{1}=\left[\begin{array}{cc}
0.1 y^{2} & -1 \\
1 & -y^{2}
\end{array}\right], A_{2}=\left[\begin{array}{cc}
0.1 y^{2} & -1 \\
-0.2172 & -y^{2}
\end{array}\right] \\
B_{1}=B_{2}=\left[\begin{array}{l}
1 \\
0
\end{array}\right], C_{1}=C_{2}=\left[\begin{array}{ll}
1 & 0
\end{array}\right] \\
h_{1}(z)=\frac{\sin y+0.2172 y}{1.217 y}, h_{2}(z)=\frac{y-\sin y}{1.2172 y},
\end{gathered}
$$

با در نظر گرفتن ساير بارامترهاى طراحى مطابق با آنجه در طراحى

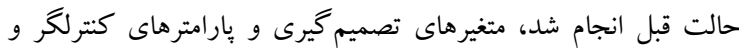

$$
\text { رويتخر فازى به صورت فيل }
$$

$$
\begin{gathered}
X_{11}=\left[\begin{array}{ll}
0.0415 & 0.0086 \\
0.0086 & 0.0707
\end{array}\right], \\
X_{22}=\left[\begin{array}{ll}
2.4376 & 0.3848 \\
0.3848 & 3.8856
\end{array}\right], \\
X_{33}=\left[\begin{array}{ll}
1.7516 & 0.0448 \\
0.0448 & 2.1999
\end{array}\right] \\
K_{1}=\left[\begin{array}{ll}
-1.1728 & 0.22032
\end{array}\right] \\
K_{2}=\left[\begin{array}{ll}
-1.1728 & 0.2032
\end{array}\right] \\
L_{1}=\left[\begin{array}{c}
0.7008 \\
-0.0671
\end{array}\right], L_{2}=\left[\begin{array}{c}
0.7124 \\
-0.1511
\end{array}\right]
\end{gathered}
$$
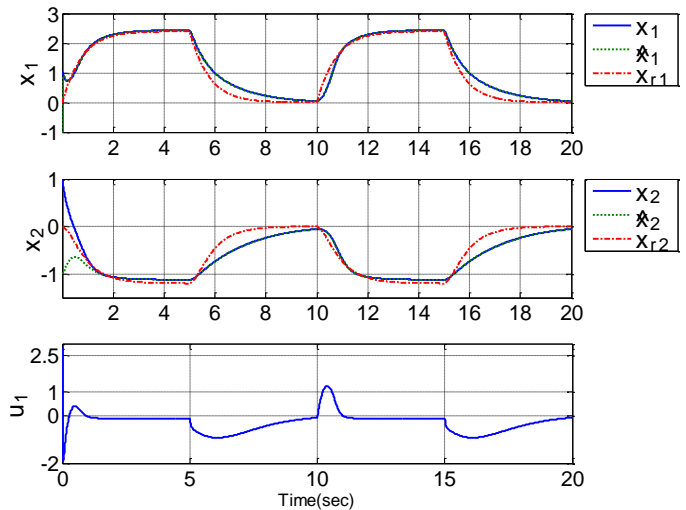

شكل ه: نتايج شبيهسازى سيستم مثال دوم

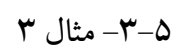$$
\text { سيستم غير خطى }
$$$$
\left\{\begin{array}{c}
\dot{x}_{1}=0.1 x_{1}^{3}-x_{2}+u \\
\dot{x}_{2}=\sin x_{1}-x_{1}^{2} x_{2} \\
y=x_{1}
\end{array}\right.
$$

را در نظر بخيريد([ra]). در اين مثال به دو روش مبتنى بر مدل تاكاگى -

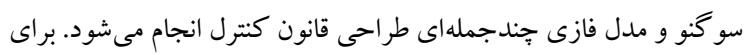

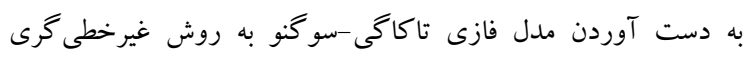

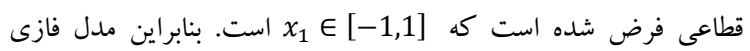

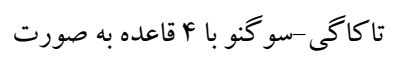

$$
\begin{gathered}
A_{1}=\left[\begin{array}{cc}
0.1 & -1 \\
1 & -1
\end{array}\right], A_{2}=\left[\begin{array}{cc}
0.1 & -1 \\
-0.217 & -1
\end{array}\right] \\
A_{3}=\left[\begin{array}{cc}
0 & -1 \\
1 & 0
\end{array}\right], A_{4}=\left[\begin{array}{cc}
0 & -1 \\
-0.217 & 0
\end{array}\right] \\
B_{1}=B_{2}=B_{3}=B_{4}=\left[\begin{array}{l}
1 \\
0
\end{array}\right], \\
C_{1}=C_{2}=C_{3}=C_{4}=\left[\begin{array}{ll}
1 & 0
\end{array}\right] \\
h_{1}(x)=x_{1}^{2} \frac{\sin x_{1}+0.217 x_{1}}{1.217 x_{1}} \\
h_{2}(x)=x_{1}^{2} \frac{x_{1}-\sin x_{1}}{1.217 x_{1}} \\
h_{3}(x)=\left(1-x_{1}^{2}\right) \frac{\sin x_{1}+0.217 x_{1}}{1.217 x_{1}}, \\
h_{4}(x)=\left(1-x_{1}^{2}\right) \frac{x_{1}-\sin x_{1}}{1.217 x_{1}}
\end{gathered}
$$

بدست مى آيد. با انتخاب بارامتر $5=5$ = مرجع به صورت

$$
A_{r}=\left[\begin{array}{cc}
-4.9 & 0 \\
1 & -1
\end{array}\right], B_{r}=\left[\begin{array}{l}
1 \\
0
\end{array}\right], C_{r}=\left[\begin{array}{ll}
1 & 0
\end{array}\right]
$$

متغيرهاى تصميم گيرى و يارامترهاى كنترلخر و رويتخر فازى به

$$
X_{11}=\left[\begin{array}{ll}
0.8676 & 0.2861 \\
0.2861 & 0.7334
\end{array}\right], X_{22}=\left[\begin{array}{ll}
0.3066 & 0.2021 \\
0.2021 & 0.6991
\end{array}\right]
$$


روزبه سليمى طارى، على معرفيانيور

Wheeled Mobile Robot With Nonholonomic Constraint," IEEE Transactions on Control Systems Technology, vol. 24, pp. 741-746, 2016.

[4] G. Wen, W. Yu, Y. Xia, X. Yu, and J. Hu, "Distributed Tracking of Nonlinear Multiagent Systems Under Directed Switching Topology: An Observer-Based Protocol," IEEE Transactions on Systems, Man, and Cybernetics: Systems, vol. 47, pp. 869-881, 2017.

[5] H. O. Wang, K. Tanaka, and M. F. Griffin, "An approach to fuzzy control of nonlinear systems: stability and design issues," IEEE Transactions on Fuzzy Systems, vol. 4, pp. 14-23, 1996.

[6] K. Tanaka and H. O. Wang, "Fuzzy regulators and fuzzy observers: a linear matrix inequality approach," in Proceedings of the 36th IEEE Conference on Decision and Control, 1997, pp. 1315-1320 vol.2.

[7] K. Tanaka, T. Ikeda, and H. O. Wang, "Fuzzy regulators and fuzzy observers: relaxed stability conditions and LMI-based designs," IEEE Transactions on Fuzzy Systems, vol. 6, pp. 250-265, 1998.

[8] M. C. M. Teixeira, E. Assunção, and H. C. Pietrobom, "On relaxed LMI-based designs for fuzzy regulators and fuzzy observers," in 2001 European Control Conference (ECC), 2001, pp. 120-125.

[9] M. C. M. Teixeira, E. Assuncao, and R. G. Avellar, "On relaxed LMI-based designs for fuzzy regulators and fuzzy observers," IEEE Transactions on Fuzzy Systems, vol. 11, pp. 613-623, 2003.

[10] T. Chung-Shi, C. Bor-Sen, and U. Huey-Jian, "Fuzzy tracking control design for nonlinear dynamic systems via T-S fuzzy model," IEEE Transactions on Fuzzy Systems, vol. 9, pp. 381-392, 2001.

[11] G. H. Chang and J. C. Wu, "Robust Tracking Control Design for Nonlinear Systems via Fuzzy Observer," in 2012 Fifth International Symposium on Computational Intelligence and Design, 2012, pp. 366-369.

[12] M. H. Asemani and V. J. Majd, "A robust Ho observer-based controller design for uncertain $\mathrm{T}-\mathrm{S}$ fuzzy systems with unknown premise variables via LMI," Fuzzy Sets and Systems, vol. 212, pp. 21-40, 2013.

[13] C. Lin, Q.-G. Wang, and T. Heng Lee, "Improvement on observer-based control for T-S fuzzy systems," Automatica, vol. 41, pp. 1651-1656, 2005.

[14] C.-S. Tseng and C.-K. Hwang, "Fuzzy observerbased fuzzy control design for nonlinear systems with persistent bounded disturbances," Fuzzy Sets and Systems, vol. 158, pp. 164-179, 2007.

[15] H. Dahmani, O. Pagès, A. E. Hajjaji, and N. Daraoui, "Observer-Based Robust Control of Vehicle Dynamics for Rollover Mitigation in Critical Situations," IEEE Transactions on

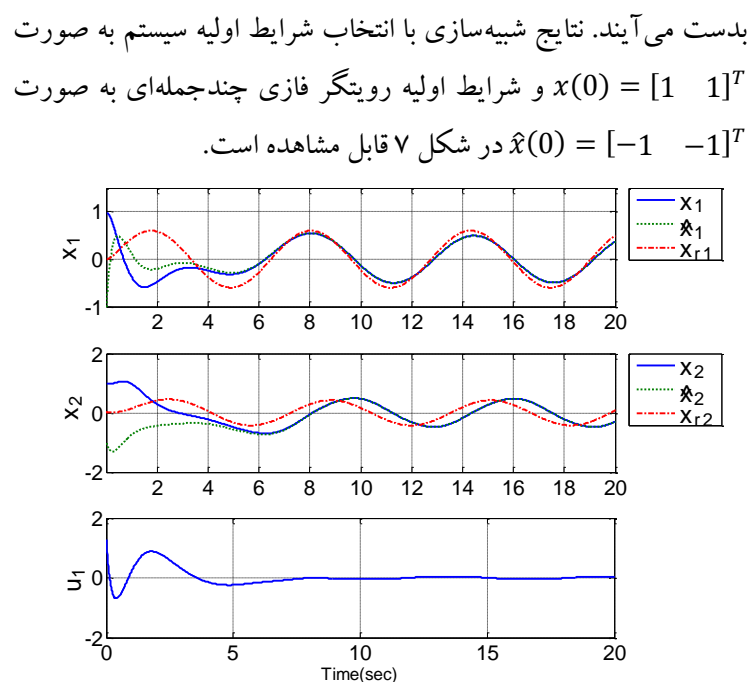

شكل V: نتايج شبيه سازى مثال سوم براى مدل فازى جِندجملهاى

همانطور كه در شكل 9 و شكل Vلاحظه مى شود در طراحى به روش

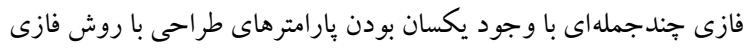

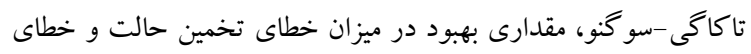

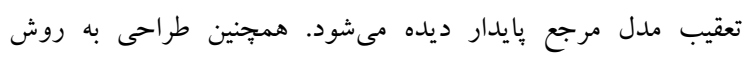

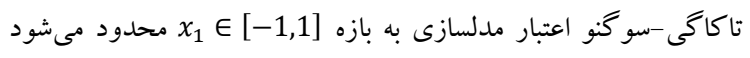
در صورتيكه در روش فازى جنّجملهاى مدلسازى و در نتيجه قانون

$$
\text { كنترل به طور جامع معتبر است. }
$$

در مقاله حاضر، قانون كنترل تعقيب مبتنى بر رويتخر براى كلاس

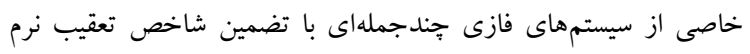

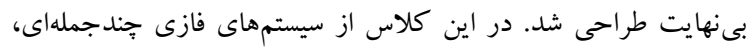

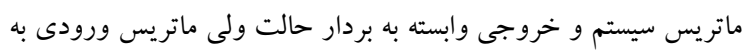

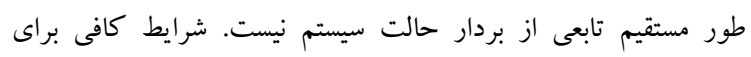

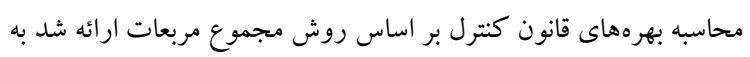

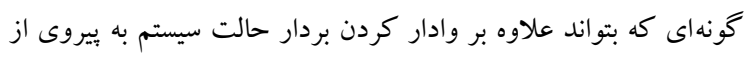

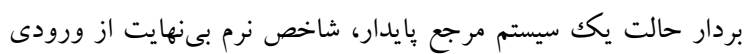

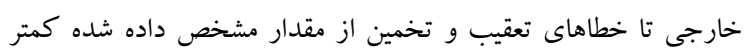

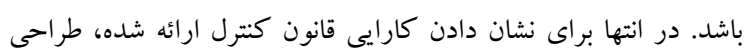

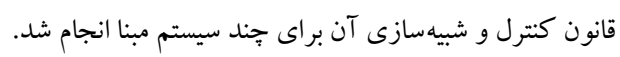
مراجع

[1] Z. Wang, R. Lu, and H. Wang, "Finite-Time Trajectory Tracking Control of a Class of Nonlinear Discrete-Time Systems," IEEE Transactions on Systems, Man, and Cybernetics: Systems, vol. 47, pp. 1679-1687, 2017.

[2] Y. F. Gao, X. M. Sun, C. Wen, and W. Wang, "Adaptive Tracking Control for a Class of Stochastic Uncertain Nonlinear Systems With Input Saturation," IEEE Transactions on Automatic Control, vol. 62, pp. 2498-2504, 2017.

[3] H. Yang, X. Fan, P. Shi, and C. Hua, "Nonlinear Control for Tracking and Obstacle Avoidance of a 
IEEE Transactions on Fuzzy Systems, vol. 17, pp. 1284-1295, 2009.

[29] K. Tanaka, H. Ohtake, T. Seo, M. Tanaka, and H. O. Wang, "Polynomial Fuzzy Observer Designs: A Sum-of-Squares Approach," IEEE Transactions on Systems, Man, and Cybernetics, Part B (Cybernetics), vol. 42, pp. 1330-1342, 2012.

[30] A. Sala, J. L. Pitarch, M. Bernal, A. Jaadari, and T. M. Guerra, "Fuzzy Polynomial observers," IFAC Proceedings Volumes, vol. 44, pp. 12772-12776, 2011.

[31] C. Liu, H. K. Lam, X. Ban, and X. Zhao, "Design of polynomial fuzzy observer-controller with membership functions using unmeasurable premise variables for nonlinear systems," Information Sciences, vol. 355-356, pp. 186-207, 2016.

[32] H. Han, J. Chen, and H. R. Karimi, "State and disturbance observers-based polynomial fuzzy controller," Information Sciences, vol. 382-383, pp. $38-59,2017$

[33] S. Prajna, A. Papachristodoulou, and W. Fen, "Nonlinear control synthesis by sum of squares optimization: a Lyapunov-based approach," in 2004 5th Asian Control Conference (IEEE Cat. No.04EX904), 2004, pp. 157-165 Vol.1.

[34] A. Papachristodoulou and S. Prajna, "A tutorial on sum of squares techniques for systems analysis," in Proceedings of the 2005, American Control Conference, 2005., 2005, pp. 2686-2700 vol. 4.

[35] F. Delmotte, M. Dambrine, S. Delrot, and S. Lalot, "Fouling detection in a heat exchanger: A polynomial fuzzy observer approach," Control Engineering Practice, vol. 21, pp. 1386-1395, 2013.

[36] H. K. Lam and H. Li, "Output-Feedback Tracking Control for Polynomial Fuzzy-Model-Based Control Systems," IEEE Transactions on Industrial Electronics, vol. 60, pp. 5830-5840, 2013.

[37] Z. Chen, B. Zhang, H. Li, and J. Yu, "Tracking control for polynomial fuzzy networked systems with repeated scalar nonlinearities," Neurocomputing, vol. 171, pp. 185-193, 2016.

[38] S. Prajna, A. Papachristodoulou, and P. A. Parrilo, "Introducing SOSTOOLS: a general purpose sum of squares programming solver," in Proceedings of the 41st IEEE Conference on Decision and Control, 2002., 2002, pp. 741-746 vol.1.

[39] S. Boyd. et. al., Linear Matrix Inequalities in System and Control Theory, in Applied Mathematics, vol. 15, PHL:SIAM, 1994

[40] C.-W. Park, C.-H. Lee, and M. Park, "Design of an adaptive fuzzy model based controller for chaotic dynamics in Lorenz systems with uncertainty," Information Sciences, vol. 147, pp. 245-266, 2002.

[41] H. Ohtake, K. Tanaka, and H. O. Wang, "Fuzzy modeling via sector nonlinearity concept," in Proceedings Joint 9th IFSA World Congress and 20th NAFIPS International Conference (Cat. No. 01TH8569), 2001, pp. 127-132.
Intelligent Transportation Systems, vol. 15, pp. 274284,2014

[16] H. Dahmani, O. Pagès, and A. E. Hajjaji, "ObserverBased State Feedback Control for Vehicle Chassis Stability in Critical Situations," IEEE Transactions on Control Systems Technology, vol. 24, pp. 636643, 2016.

[17] P. Bergsten, R. Palm, and D. Driankov, "Observers for Takagi-Sugeno fuzzy systems," IEEE Transactions on Systems, Man, and Cybernetics, Part B (Cybernetics), vol. 32, pp. 114-121, 2002.

[18] L. Li, S. X. Ding, J. Qiu, Y. Yang, and D. Xu, "Fuzzy Observer-Based Fault Detection Design Approach for Nonlinear Processes," IEEE Transactions on Systems, Man, and Cybernetics: Systems, vol. PP, pp. 1-12, 2016.

[19] L. Li, S. X. Ding, J. Qiu, and Y. Yang, "Real-Time Fault Detection Approach for Nonlinear Systems and its Asynchronous T-S Fuzzy Observer-Based Implementation," IEEE Transactions on Cybernetics, vol. 47, pp. 283-294, 2017.

[20] Y. Wu, J. Dong, X. J. Li, and G. H. Yang, "A new fault detection observer scheme for T-S fuzzy systems with unmeasurable variables," in 2016 12th World Congress on Intelligent Control and Automation (WCICA), 2016, pp. 120-125.

[21] Y. Yang, S. X. Ding, and L. Li, "On observer-based fault detection for nonlinear systems," Systems \& Control Letters, vol. 82, pp. 18-25, 2015.

[22] L. Li, S. X. Ding, Y. Yang, and Y. Zhang, "Robust fuzzy observer-based fault detection for nonlinear systems with disturbances," Neurocomputing, vol. 174, Part B, pp. 767-772, 2016.

[23] T. Agustinah, A. Jazidie, M. Nuh, and D. Haiping, "Fuzzy tracking control design using observer-based stabilizing compensator for nonlinear systems," in 2010 International Conference on System Science and Engineering, 2010, pp. 275-280.

[24] K. Tanaka, H. Yoshida, H. Ohtake, and H. O. Wang, "A Sum of Squares Approach to Stability Analysis of Polynomial Fuzzy Systems," in 2007 American Control Conference, 2007, pp. 4071-4076.

[25] K. Tanaka, H. Yoshida, H. Ohtake, and H. O. Wang, "Stabilization of Polynomial Fuzzy Systems via a Sum of Squares Approach," in 2007 IEEE 22nd International Symposium on Intelligent Control, 2007, pp. 160-165.

[26] K. Tanaka, H. Ohtake, and H. O. Wang, "Guaranteed Cost Control of Polynomial Fuzzy Systems via a Sum of Squares Approach," IEEE Transactions on Systems, Man, and Cybernetics, Part B (Cybernetics), vol. 39, pp. 561-567, 2009.

[27] K. Tanaka, H. Yoshida, H. Ohtake, and H. O. Wang, "A Sum-of-Squares Approach to Modeling and Control of Nonlinear Dynamical Systems With Polynomial Fuzzy Systems," IEEE Transactions on Fuzzy Systems, vol. 17, pp. 911-922, 2009.

[28] A. Sala and C. Arino, "Polynomial Fuzzy Models for Nonlinear Control: A Taylor Series Approach," 